\title{
Effect of dilute acetic acid hydrolysis on xylo-oligosaccharides production and inhibitory effect of cellulolytic enzyme lignin from poplar
}

Peiyao Wena,b,c†,Tian Zhang ${ }^{\mathrm{a} \dagger}$, Liting Wei ${ }^{\mathrm{a}}$, Jinye Wang ${ }^{\mathrm{a}}$, Arthur J. Ragauskas ${ }^{\mathrm{d}, \mathrm{e}}$, Ying Zhanga, Yong $\mathrm{Xu}^{\mathrm{b}, \mathrm{c}}$, Junhua Zhanga,b, $*$

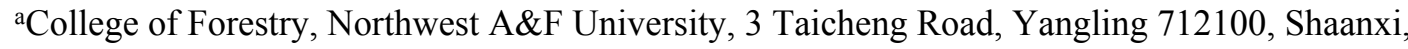
China

bJiangsu Co-Innovation Center of Efficient Processing and Utilization of Forest Resources, College of Chemical Engineering, Nanjing Forestry University, 159 Longpan Road, Nanjing 210037, China

'Key Laboratory of Forestry Genetics \& Biotechnology (Nanjing Forestry University), Ministry of Education, 159 Longpan Road, Nanjing 210037, China

${ }^{\mathrm{d} D e p a r t m e n t}$ of Chemical and Biomolecular Engineering, University of Tennessee, Knoxville, TN 37996-2200, USA

eJoint Institute for Biological Sciences, Oak Ridge National Laboratory (ORNL), Oak Ridge, TN 37831, USA

*Corresponding author. Tel.: +86-13892883052

E-mail address: junhuazhang@,nwafu.edu.cn

$\dagger$ Peiyao Wen and Tian Zhang contributed equally.

- $\quad$ Number of pages: 11

- $\quad$ Number of tables: 4

- $\quad$ Number of figures: 5

Contents

Page

Table S1 FT-IR of CELs samples .2

Table S2 The relative distance between O-H and C-H $\left(\mathrm{cm}^{-1}\right)$ in FT-IR .................................

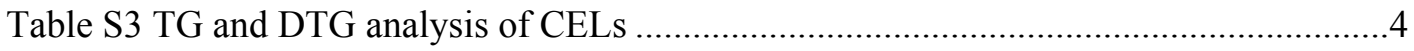

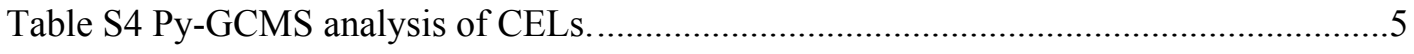

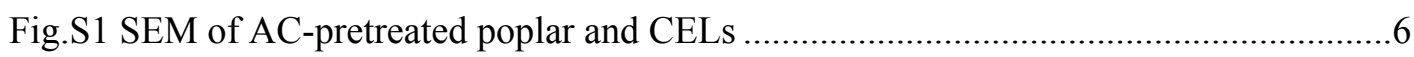

Fig.S2 The remaining filter paper activity in the enzymatic hydrolysis of $2 \%$ poplar...........

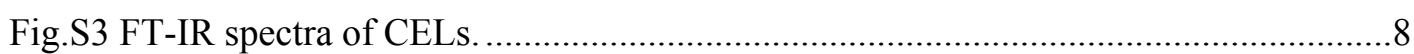

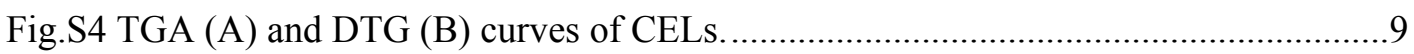

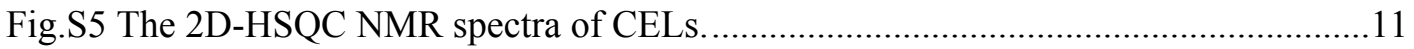


Table S1 FT-IR of CELs samples

\begin{tabular}{cc}
\hline Wavenumber $\left(\mathrm{cm}^{-1}\right)$ & Band origin \\
\hline 1660 & Aryl ring stretch, symmetric \\
1591 & Aromatic skeletal vibrations \\
1513 & Aromatic skeletal vibrations \\
1463 & C-H bending in $-\mathrm{CH}_{3}$ and $-\mathrm{CH}_{2}-$ \\
1420 & Aromatic ring vibrations and C-H in plane \\
1327 & deformation \\
1272 & Gyringyl ring and C-O stretching vibration \\
1228 & Guaiacyl ring and C-O stretching vibration \\
1124 & Aromatic C-H deformation in Syringyl \\
1031 & C-O-C stretching \\
\hline
\end{tabular}


Table S2 The relative distance between $\mathrm{O}-\mathrm{H}$ and $\mathrm{C}-\mathrm{H}\left(\mathrm{cm}^{-1}\right)$ in FT-IR

\begin{tabular}{cccc}
\hline Samples & $\begin{array}{c}\text { Wavenumber of CH } \\
\left(\mathrm{cm}^{-1}\right)\end{array}$ & $\begin{array}{c}\text { Wavenumber of OH } \\
\left(\mathrm{cm}^{-1}\right)\end{array}$ & $\begin{array}{c}\text { Distance between O-H } \\
\text { and C-H }\left(\mathrm{cm}^{-1}\right)\end{array}$ \\
\hline BM-CEL & 2935.732 & 3407 & 471.268 \\
$160-C E L$ & 2937.661 & 3420.28 & 482.619 \\
$170-C E L$ & 2938.625 & 3420.28 & 481.655 \\
$180-C E L$ & 2938.143 & 3420.28 & 482.137 \\
\hline
\end{tabular}


Table S3 TG and DTG analysis of CELs

\begin{tabular}{cccc}
\hline Lignin & $\mathrm{T}_{\max }\left({ }^{\circ} \mathrm{C}\right)$ & $\mathrm{DTG}_{\max }(\% / \mathrm{min})$ & Residue (\%) \\
\hline BM-CEL & 307 & 0.41 & 30.89 \\
160-CEL & 314 & 0.47 & 36.19 \\
170-CEL & 311 & 0.41 & 39.96 \\
180-CEL & 323 & 0.40 & 42.19 \\
\hline
\end{tabular}

$T_{\max }$ : The peak temperature of DTG curve. This is where the mass loss occurs at the highest rate under the DTG conditions used. DTG $_{\max }$ : The maximum decomposition rate. 
Table S4 Py-GCMS analysis of CELs.

\begin{tabular}{|c|c|c|c|c|c|c|c|}
\hline Label & Formula & Compound & Origin & $\begin{array}{l}\text { BM- } \\
\text { CEL }\end{array}$ & $\begin{array}{l}160- \\
\text { CEL }\end{array}$ & $\begin{array}{l}170- \\
\mathrm{CEL}\end{array}$ & $\begin{array}{l}180- \\
\text { CEL }\end{array}$ \\
\hline 1 & $\mathrm{C}_{6} \mathrm{H}_{6} \mathrm{O}$ & Phenol & $\mathrm{H}$ & 7.67 & 6.45 & 4.61 & 4.46 \\
\hline 2 & $\mathrm{C}_{7} \mathrm{H}_{8} \mathrm{O}_{2}$ & Phenol, 4-methoxy & $\mathrm{H}$ & 1.81 & 5.01 & 7.09 & 7.77 \\
\hline 3 & $\mathrm{C}_{8} \mathrm{H}_{10} \mathrm{O}_{2}$ & Phenol, 2-methoxy-4-methyl- & G & 1.64 & 1.04 & 1.82 & 2.24 \\
\hline 4 & $\mathrm{C}_{9} \mathrm{H}_{12} \mathrm{O}_{2}$ & Phenol, 4-ethyl-2-methoxy & G & 0.54 & 0.48 & 0.75 & 0.86 \\
\hline 5 & $\mathrm{C}_{9} \mathrm{H}_{10} \mathrm{O}_{2}$ & 2-Methoxy-4-vinylphenol & FA & 2.88 & 4.05 & 4.02 & 3.86 \\
\hline 6 & $\mathrm{C}_{9} \mathrm{H}_{10} \mathrm{O}$ & 3-Methyl-2, 3-dihydro-benzofuran & & 0.23 & - & - & - \\
\hline \multirow[t]{2}{*}{7} & $\mathrm{C}_{8} \mathrm{H}_{10} \mathrm{O}_{3}$ & Phenol, 2, 6-dimethoxy & S & 5.16 & 11.75 & 15.61 & 16.35 \\
\hline & $\mathrm{C}_{10} \mathrm{H}_{14} \mathrm{O}_{2}$ & Phenol, 2-methoxy-4-propyl- & G & - & - & 0.31 & 0.32 \\
\hline 8 & $\mathrm{C}_{10} \mathrm{H}_{12} \mathrm{O}_{2}$ & Phenol, 2-methoxy-4-(1-propenyl) & FA & 0.35 & 0.34 & - & - \\
\hline 9 & $\mathrm{C}_{8} \mathrm{H}_{8} \mathrm{O}_{3}$ & Vanillin & G & 0.52 & 0.46 & - & - \\
\hline 10 & $\mathrm{C}_{9} \mathrm{H}_{12} \mathrm{O}_{3}$ & 1,2,4-Trimethoxybenzene & $\mathrm{G}$ & 3.33 & 1.94 & 2.28 & 3.23 \\
\hline 11 & $\mathrm{C}_{10} \mathrm{H}_{12} \mathrm{O}_{2}$ & Phenol, 2-methoxy-4-(1-propenyl) & G & 3.13 & 2.28 & 3.18 & 2.55 \\
\hline 12 & $\mathrm{C}_{9} \mathrm{H}_{10} \mathrm{O}_{3}$ & $\begin{array}{l}\text { Ethanone, } \\
\text { 1-(4-hydroxy-3-methoxyphenyl)- }\end{array}$ & G & 0.56 & 0.25 & 0.32 & 0.33 \\
\hline 13 & $\mathrm{C}_{10} \mathrm{H}_{14} \mathrm{O}_{3}$ & Benzene, 1, 2, 3-trimethoxy-5-methyl- & S & 0.60 & 0.79 & 1.08 & 1.03 \\
\hline 14 & $\mathrm{C}_{10} \mathrm{H}_{12} \mathrm{O}_{3}$ & $\begin{array}{l}\text { 2-Propanone, } \\
\text { 1-(4-hydroxy-3-methoxyphenyl)- }\end{array}$ & G & 0.23 & 0.33 & 0.42 & 0.43 \\
\hline 15 & $\mathrm{C}_{10} \mathrm{H}_{12} \mathrm{O}_{3}$ & 3', 5'-Dimethoxyacetophenone & S & 3.78 & 5.57 & 5.33 & 4.34 \\
\hline 16 & $\mathrm{C}_{11} \mathrm{H}_{14} \mathrm{O}_{3}$ & Phenol, 2,6-dimethoxy-4-(2-propenyl) & S & 1.13 & 0.77 & 0.90 & 0.78 \\
\hline 17 & $\mathrm{C}_{11} \mathrm{H}_{14} \mathrm{O}_{3}$ & Phenol, 2, 6-dimethoxy-4-(2-propenyl) & S & 0.57 & 0.46 & 0.53 & 0.45 \\
\hline 18 & $\mathrm{C}_{9} \mathrm{H}_{10} \mathrm{O}_{4}$ & $\begin{array}{l}\text { Benzaldehyde, } \\
\text { 5-dimethoxy }\end{array}$ & $\mathrm{S}$ & 1.02 & 0.63 & 0.74 & 0.67 \\
\hline 19 & $\mathrm{C}_{10} \mathrm{H}_{12} \mathrm{O}_{3}$ & $\begin{array}{l}\text { 4-((1E)-3-Hydroxy-1-propenyl)-2-meth } \\
\text { oxyphenol }\end{array}$ & $\mathrm{G}$ & 0.82 & 0.48 & - & - \\
\hline 20 & $\mathrm{C}_{11} \mathrm{H}_{14} \mathrm{O}_{3}$ & Phenol, 2, 6-dimethoxy-4-(2-propenyl)- & S & 5.35 & 3.91 & 4.39 & 3.74 \\
\hline 21 & $\mathrm{C}_{10} \mathrm{H}_{12} \mathrm{O}_{4}$ & $\begin{array}{l}\text { Ethanone, 1-(4-hydroxy-3, } \\
\text { 5-dimethoxyphenyl)- }\end{array}$ & $\mathrm{S}$ & 0.80 & 0.49 & 0.57 & 0.56 \\
\hline 22 & $\mathrm{C}_{10} \mathrm{H}_{12} \mathrm{O}_{3}$ & $\begin{array}{l}\text { 4-((1E)-3-Hydroxy-1-propenyl)-2-meth } \\
\text { oxyphenol }\end{array}$ & G & 5.77 & 5.27 & 2.81 & 1.70 \\
\hline 23 & $\mathrm{C}_{10} \mathrm{H}_{12} \mathrm{O}_{5}$ & $\begin{array}{l}\text { 3, 5-Dimethoxy-4-hydroxyphenyl acetic } \\
\text { acid }\end{array}$ & S & 1.16 & 1.28 & 1.09 & 1.02 \\
\hline 24 & $\mathrm{C}_{10} \mathrm{H}_{12} \mathrm{O}_{4}$ & $\begin{array}{l}\text { Ethanone, } \\
\text { 1-(4-hydroxy-3,5-dimethoxyphenyl)- }\end{array}$ & $\mathrm{S}$ & 0.27 & 0.49 & 0.21 & 0.22 \\
\hline 25 & & & $\mathrm{H} \%$ & 9.48 & 11.46 & 11.70 & 12.23 \\
\hline 26 & & & $\mathrm{G} \%$ & 16.54 & 12.53 & 11.89 & 11.66 \\
\hline 27 & & & $\mathrm{~S} \%$ & 19.84 & 26.14 & 30.45 & 29.16 \\
\hline 28 & & & FA\% & 3.23 & 4.39 & 4.02 & 3.86 \\
\hline 29 & & & $\mathrm{~S} / \mathrm{G}$ & 1.20 & 2.09 & 2.56 & 2.50 \\
\hline
\end{tabular}



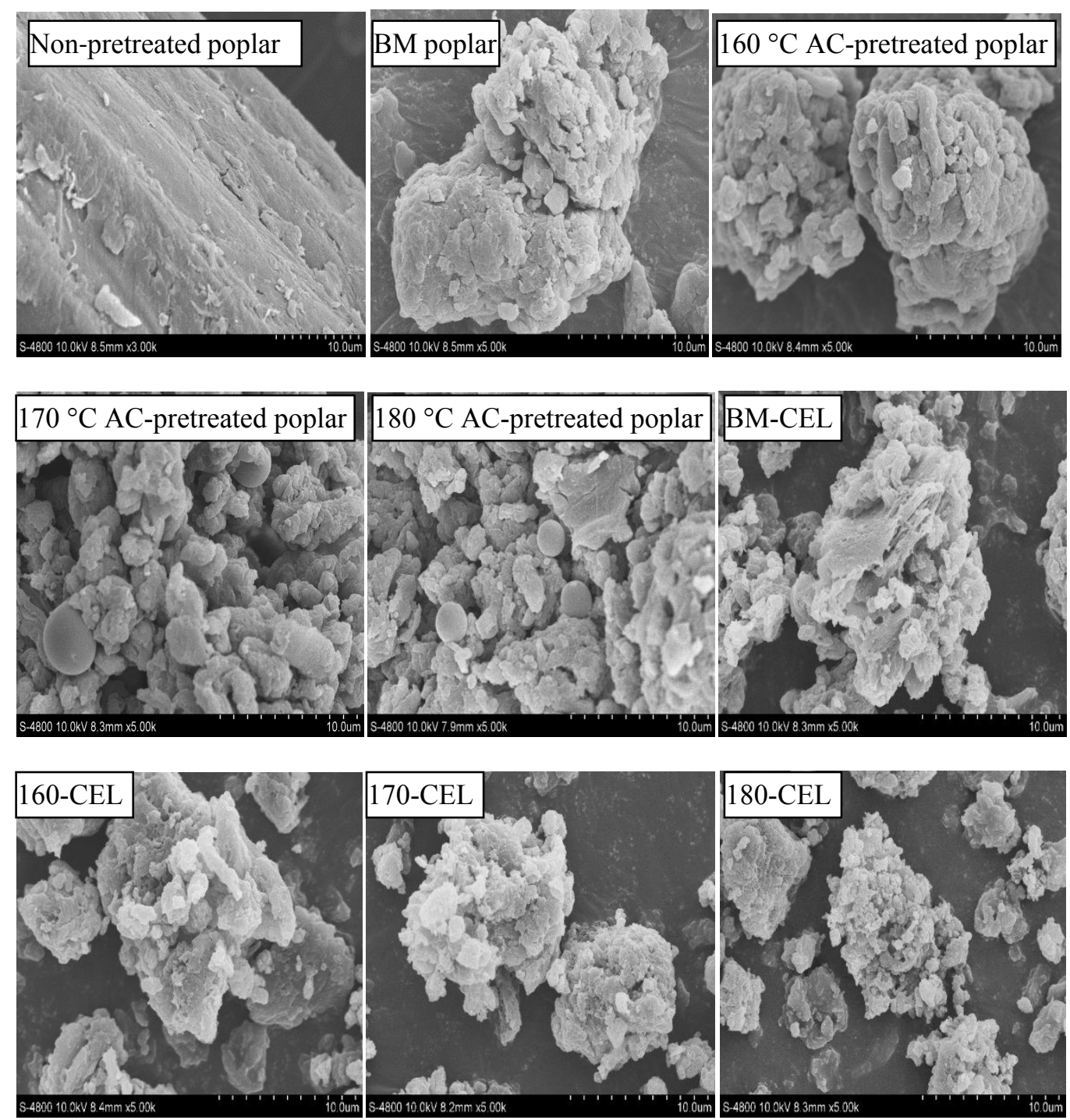

Fig.S1 SEM of AC-pretreated poplar and CELs 


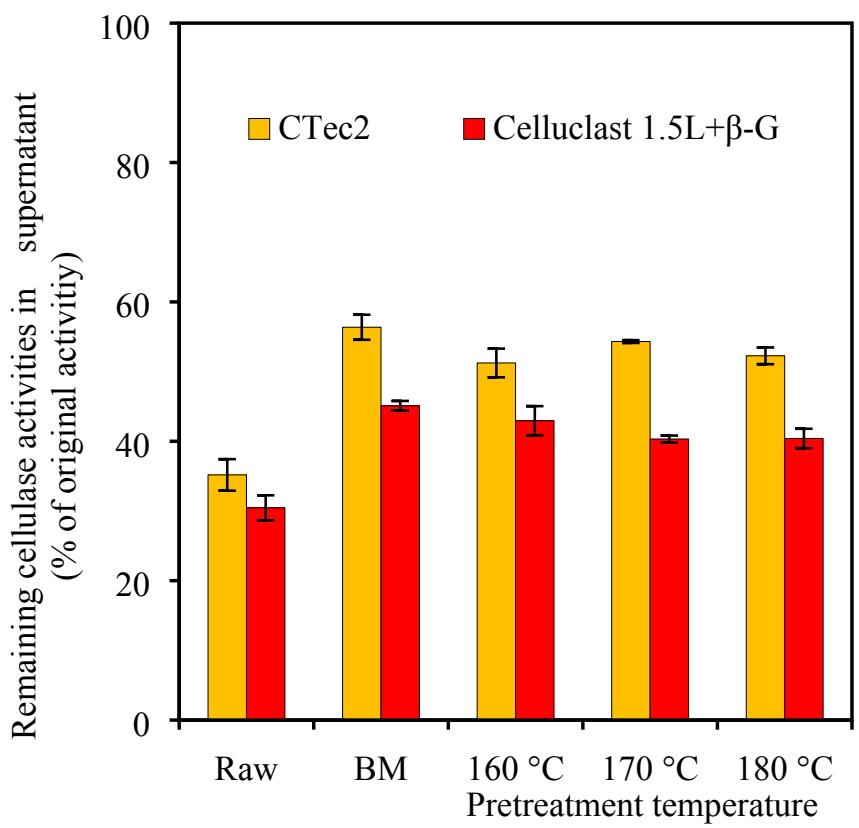

Fig.S2 The remaining filter paper activity in the enzymatic hydrolysis of $2 \%$ poplar by Celluclast 1.5L (20 FPU/g DM)/ $\beta-G(500$ nakt/g DM) or CTec2 (20 FPU/g DM) at $50{ }^{\circ} \mathrm{C}$ and $\mathrm{pH} 5.0$ for $72 \mathrm{~h}$. 

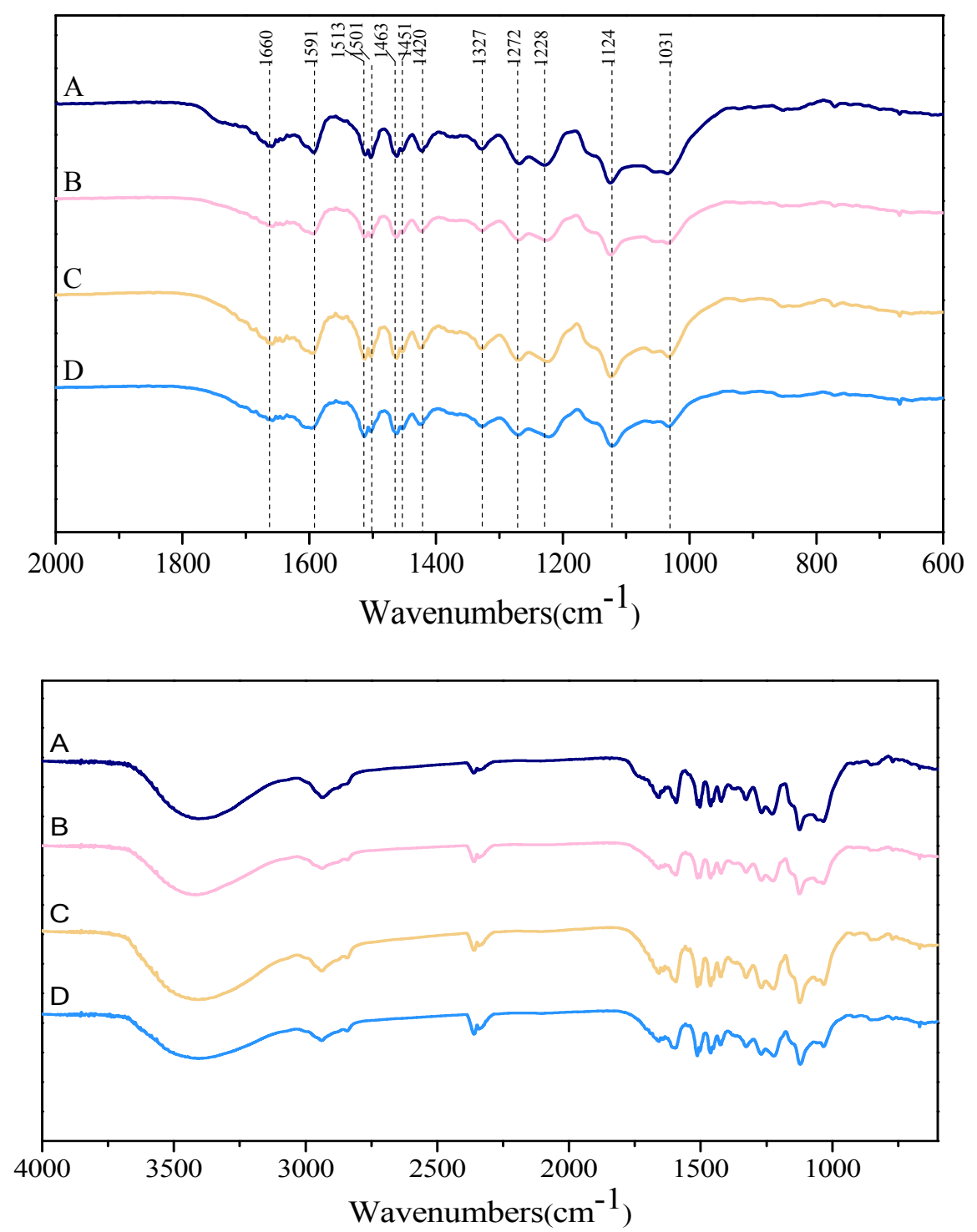

Fig.S3 FT-IR spectra of BM-CEL (A), 160-CEL (B), 170-CEL (C) and 180-CEL (D). 

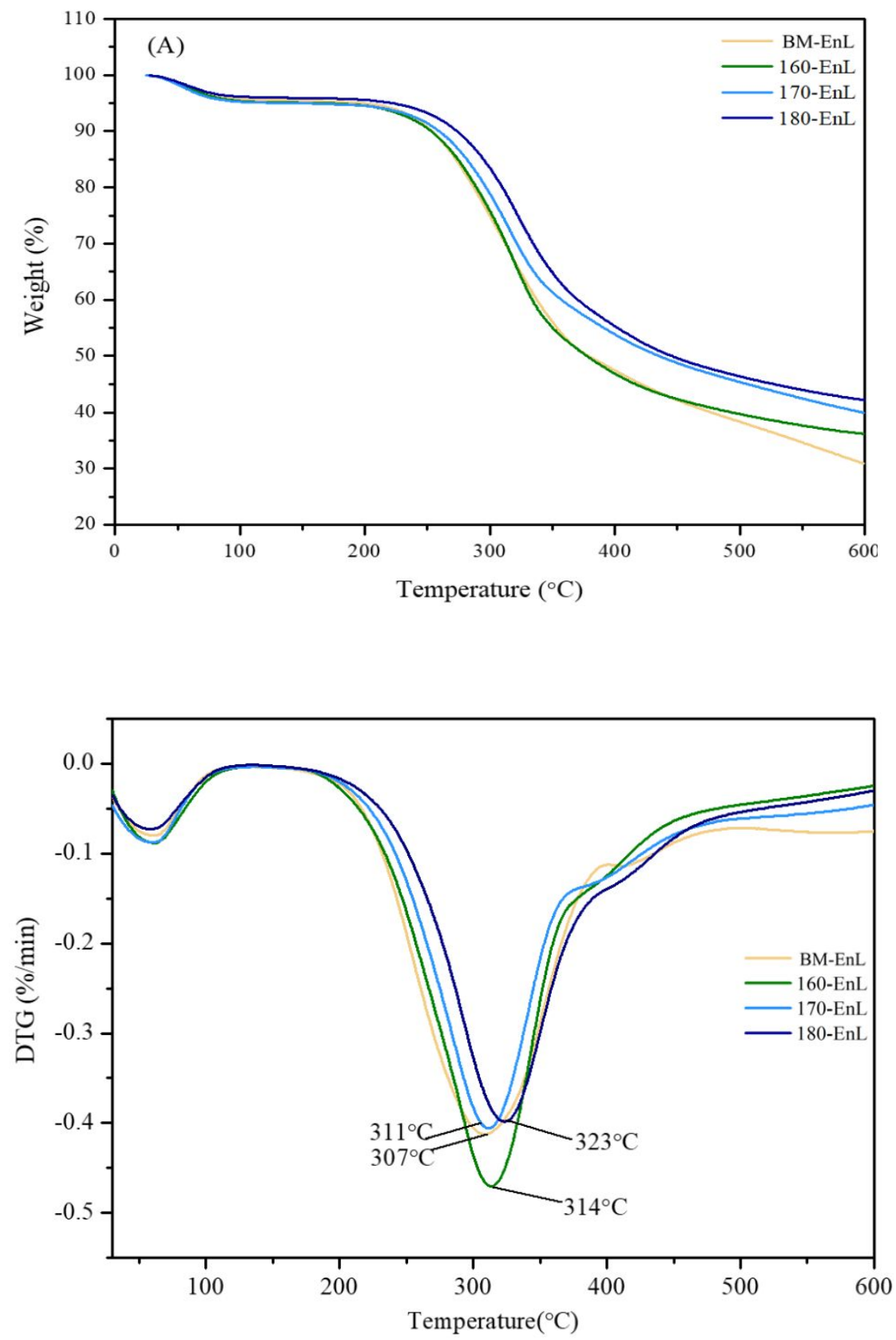

Fig.S4 TGA (A) and DTG (B) curves of CELs. 

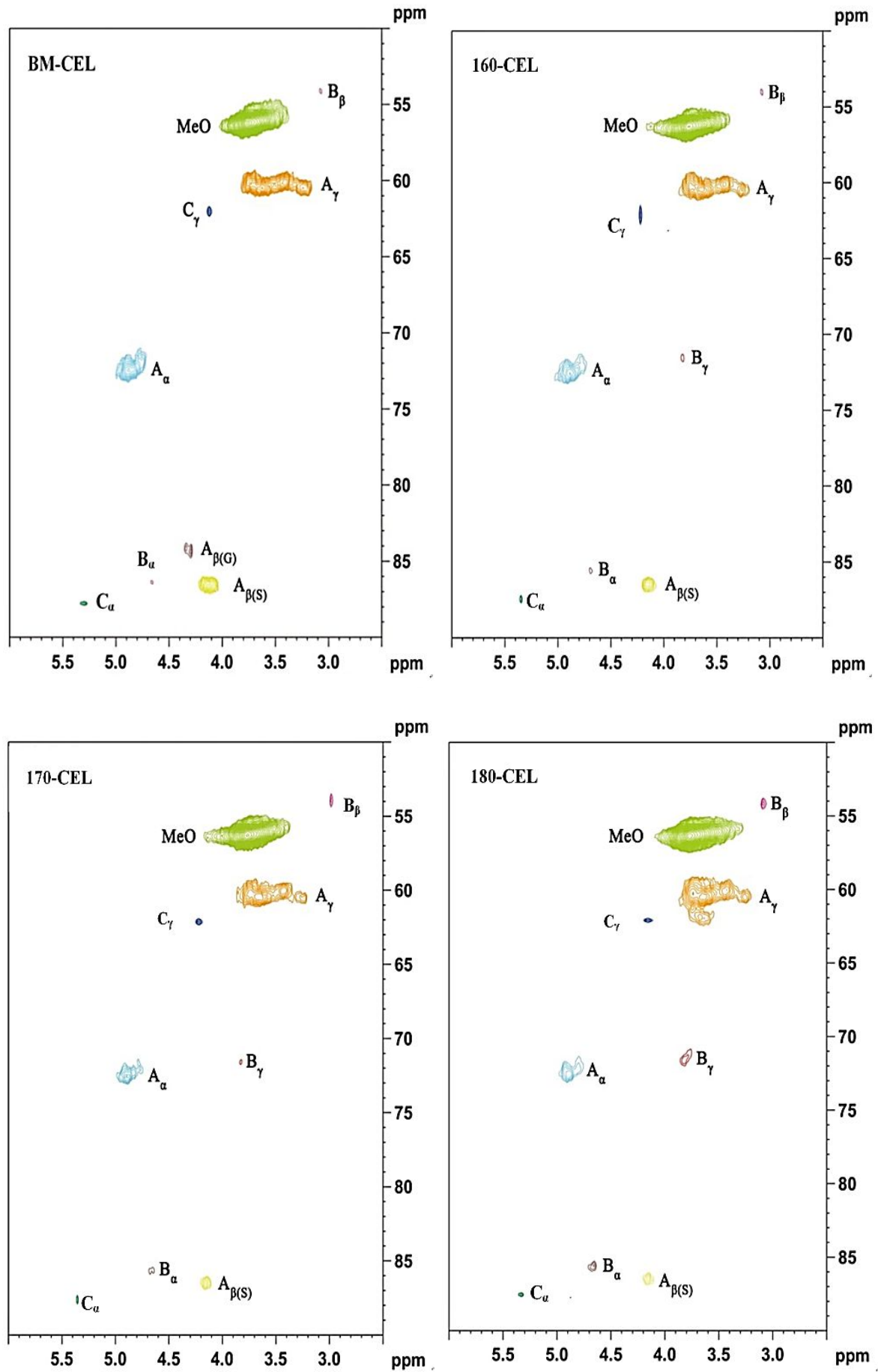

(a): Side-chain region 

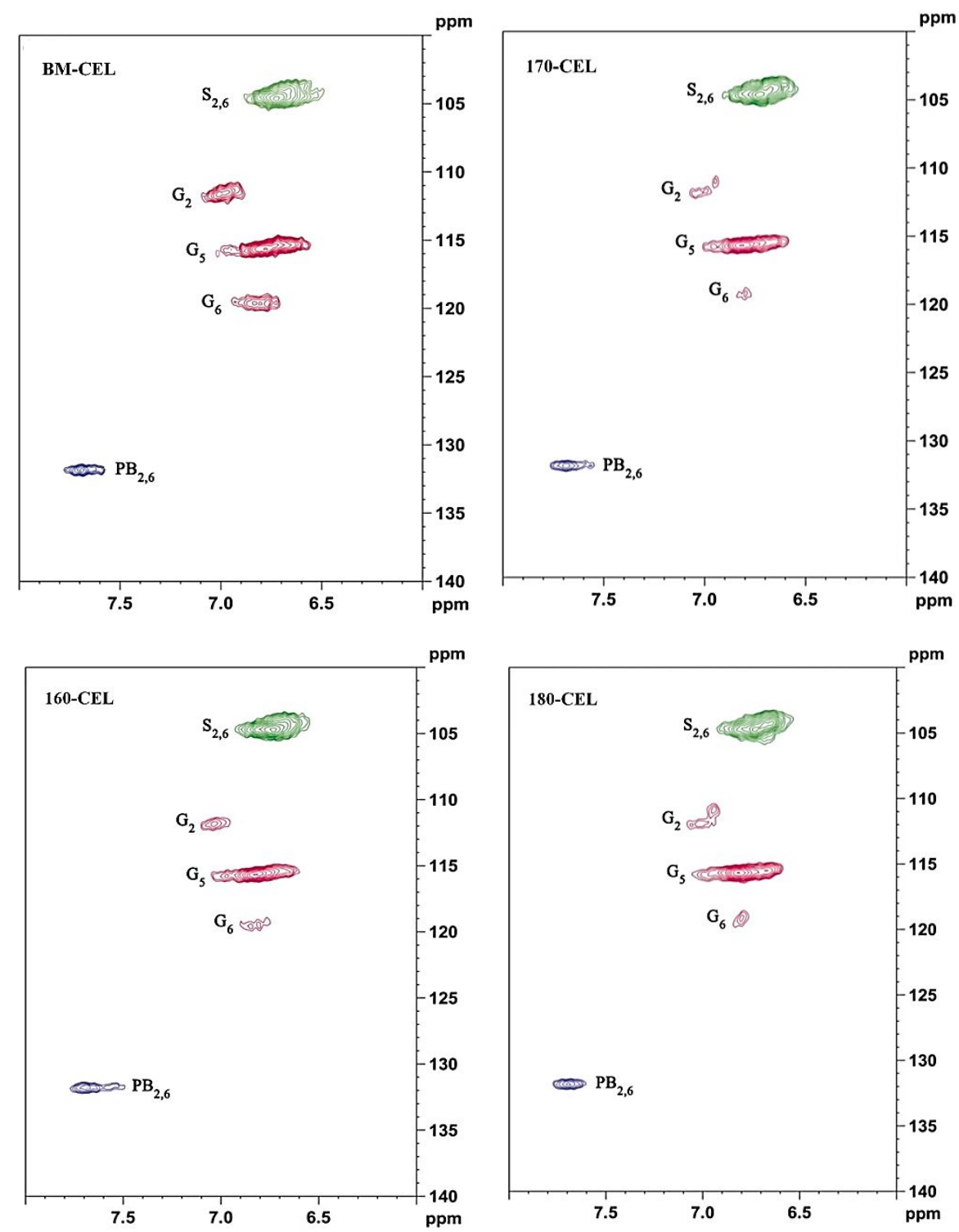

(b): aromatic region

Fig.S5 The 2D-HSQC NMR spectra of CELs. 